\title{
Modeling and Simulation of Space Self-organizing Network based on OPNET
}

\author{
Lei Yang a, Shuai Wu ${ }^{b}$, Chengguang Fan ${ }^{c}$, Jianming Guo ${ }^{d}$, Pengfei Liu ${ }^{e}$, \\ Xin Song ${ }^{f}$
}

\author{
College of Aerospace Science and Engineering, National University of Defense Technology, \\ Changsha 410073, China. \\ accraftyang@163.com, bwushuai1632009@163.com, chengguangfannudt@163.com, \\ dgjm08110@hotmail.com, ${ }^{\mathrm{e}}$ pengfeiliu_nudt@sina.com, ${ }^{\mathrm{f}}$ sonnyxin@126.com
}

Keywords: Micro-Nano Satellite, Cluster Flying, Space Self-Organizing Network, OPNET, Modeling, Simulation.

\begin{abstract}
A scheme of space self-organizing network based on TDMA (Time Division Multiple Access) is proposed for cooperative requirement in cluster flying micro-nano satellites. Network level, node level and process level models are created based on OPNET. Simulation experiments such as nodes autonomous networking, nodes autonomous joining, nodes autonomous exiting and network reorganizing are carried out and the key indexes such as networking time are tested. The scheme has been used in the cluster flying TianTuo-3 satellites developed by National Defense University of Science and Technology and on-orbit networking tests have been successfully carried out. This paper can provide a reference for the design and simulation of space self-organizing network.
\end{abstract}

\section{Introduction}

Space mission carried out by cluster flying spacecrafts has become one of the hot research areas [1]. Compared with the traditional satellites, cluster flying small satellites have more advantages in terms of development cost, risk control, overall reliability, flexibility and maintainability. In space missions that require longer measurement baseline such as interferometry, cluster flying micro satellites are more acceptable [2,3]. Cluster flying micro satellites require a reliable and efficient network to share information and cooperate with each other in order to complete a specific task collaboratively $[4,5]$. Due to limited ground control scope, satellites should have the ability of autonomous networking. The network should also be able to reorganize, which means that the remaining nodes can be reconstructed to form a new network even when some nodes are broken.

Space self-organizing network refers to wireless information network in spacecrafts with the ability of self-discovery, self-configuration and self-healing. In recent years, the study of space selforganizing network has attracted the attention of scholars both at home and abroad. As the micronano satellites have strict limits on the volume, power and weight, cluster flying micro-nano satellites are not the same as others in terms of network application requirements and network node design constraints. At present, there is no mature standard of space self-organizing network for cluster flying micro-nano satellites and corresponding products. At the same time, due to the high cost of spacebased system, it is unrealistic to build one on-orbit system to carry out space self-organizing network experiments. Therefore, the ground simulation experiment is a feasible way to study space selforganizing network for cluster flying micro-nano satellites [6].

In this paper, a new method of space self-organizing network based on TDMA is proposed for cluster flying micro-nano satellites. Protocol implementation and simulation tests are carried out with OPNET. RF (Radio Frequency) SoC(System on a Chip) is used for programming and physical tests. On-orbit tests of space self-organizing network payloads have been carried out in the cluster flying TianTuo-3 satellites (Fig. 1), which consist of 6 satellites devided in to two groups. 


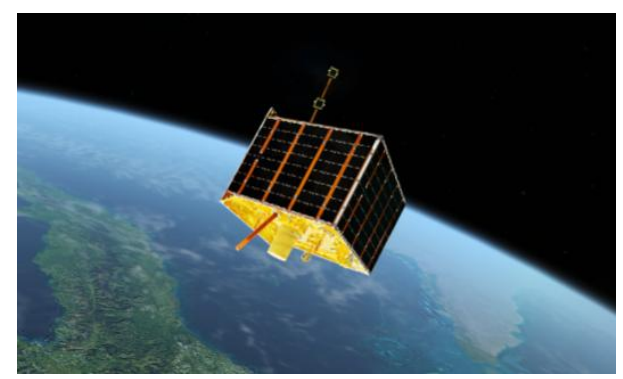

a) Main satellite and 2 femto satellites

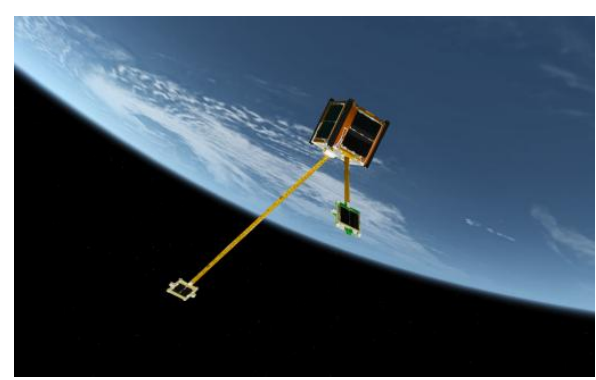

b) Phonesat and 2 femto satellites

Fig. 1 Cluster flying of TianTuo-3 satellites (6 satellites, divided into 2 groups)

\section{Design of Space Self-organizing Network}

TDMA does not need priori information and high hardware requirements and it is convenient to realize and suitable for micro-nano satellite. The design of space self-organizing network uses TDMA to fulfill multi-node communication. The network nodes work in 3 modes: master mode, slave mode and listening mode. Listening mode is a transition state. In this state, nodes negotiate to determine whether to work in master mode or slave mode. Node in the master mode is responsible for coordinating the whole network.

For presentation convenience, the basic time period for communication management is called a frame, which is a time concept different from a data frame. A frame is divided into time slots with equal length. Nodes send data in its allocated time slots. The frame structure is shown in Fig. 2.

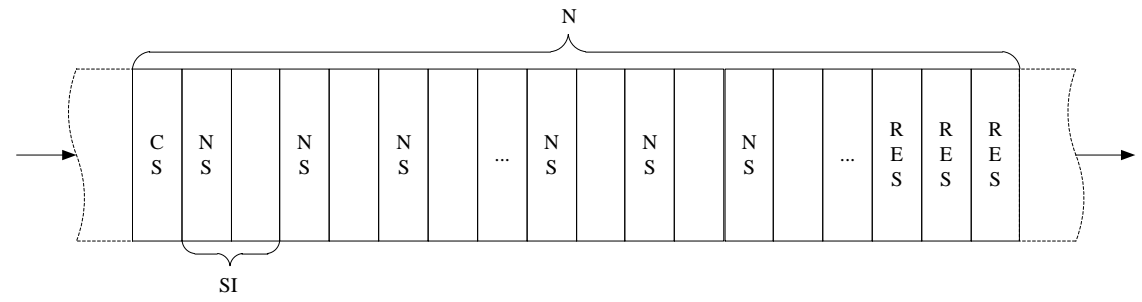

Fig. 2 Frame structure

The symbols in the frame structure are shown in the following table.

Table 1. Description of time slot symbols

\begin{tabular}{|c|c|c|}
\hline Code & Name & Destination \\
\hline $\mathrm{N}$ & Number & The total number of slots in a frame \\
\hline $\mathrm{Q}$ & Quantity & Number of nodes that have entered the network \\
\hline CS & Control Slot & $\begin{array}{c}\text { In this time slot, the master node transmits a control packet, } \\
\text { and the control packet includes information for each slot }\end{array}$ \\
\hline NS & Node Starting & The slot that node starts transmitting \\
\hline SI & Select Interval & $\begin{array}{c}\text { The number of time slots that the same node can use } \\
\text { continuously }\end{array}$ \\
\hline RES & Reserved Slots & Reserved for newly added node \\
\hline
\end{tabular}

As shown above, each frame is divided into $\mathrm{N}$ time slots, where the first slot is used for CS and the last M slots are reserved for RES. Therefore, only (N-M-1) slots are actually available for network communication within each frame. These slots are allocated to $\mathrm{Q}$ nodes evenly. So SI can be calculated as Equation 1.

$$
S I=(N-M-1) / Q \text {. }
$$

The master node counts the nodes in the network and broadcasts the list of nodes in the network. NS is calculated according to the position of the node in the list, as shown in Equation 2.

$$
N S=1+i *(Q * S I), i=0,1, \ldots,(N-M-1) / Q \text {. }
$$

The autonomous networking process is shown in Fig. 3. After powering up, each node firstly enters the listening mode. In the listening mode, if the node receives two control packets continuously, it indicates that the network already has a master node. Then the node broadcasts network access packets and switches to the slave mode after receiving permission packet within the specified time. 
However, if the node does not receive any control packet within the specified time, it is assumed that there is no master node in the network and the node switches itself to the master mode. In the master mode, the node broadcasts control packets in CS to coordinate the whole network nodes.

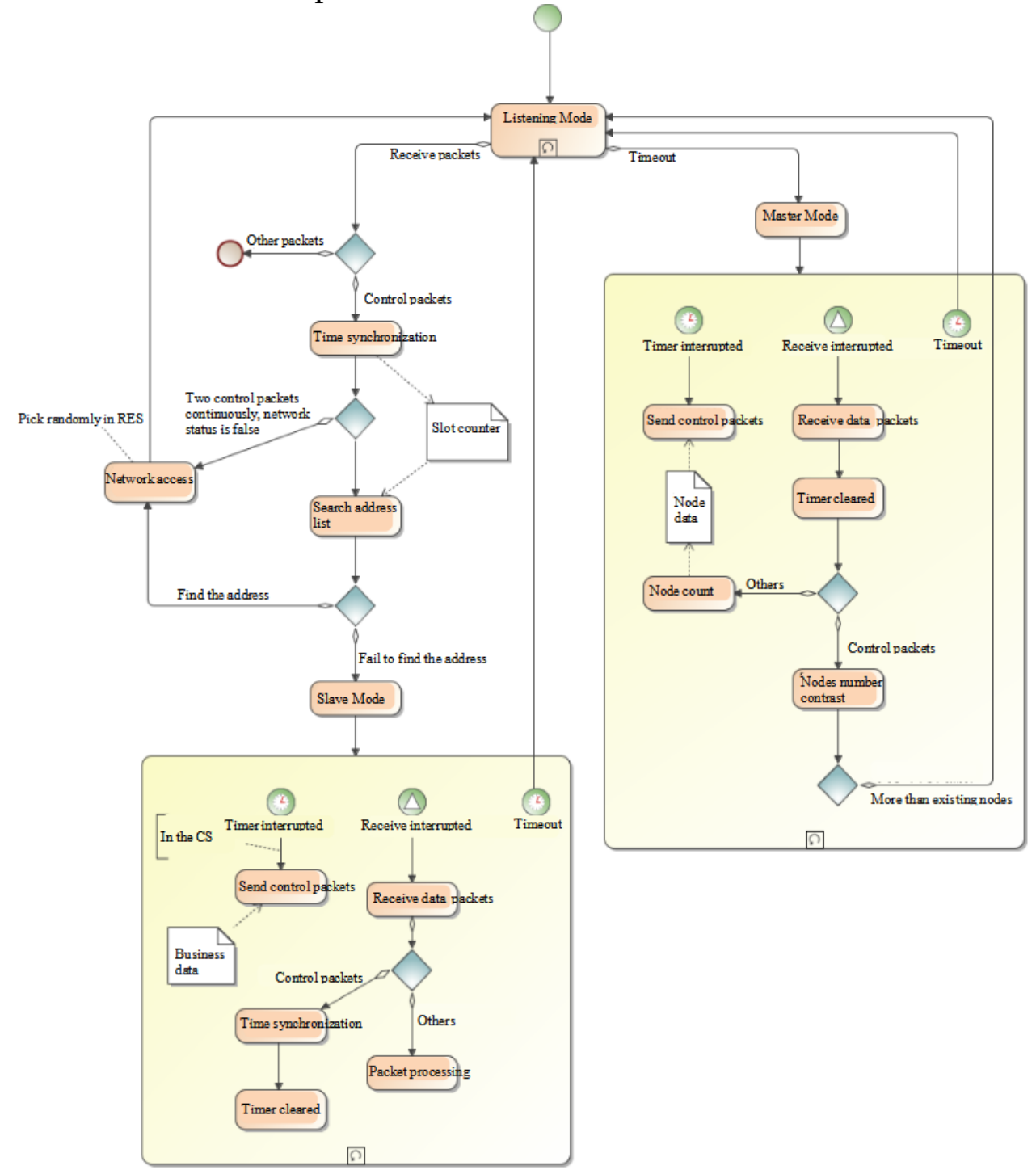

Fig. 3 Autonomous networking process

\section{Modeling of Space Self-organizing Network}

OPNET is an excellent network simulation platform, which can accurately analyze the performance and behavior of complex networks, and has been widely used in communication, aerospace, network system integration and so on. OPNET uses three-layer modeling composed of network layer, node layer and process layer. As the configuration of micro-nano satellites cluster is relatively stable, the movement of the node movement is simplified to flat motion instead of threedimension.

Node model is firstly built. It includes an antenna, a sending module, a receiving module, a processing module, as shown in Fig. 4. As the packets are sent in an orderly manner under TDMA and assuming that the data generation rate is less than the transmission rate, no queue module is added to the node model. Assuming that the distance between the aircraft is less than $50 \mathrm{~km}$, antenna module with omni direction, $1 \mathrm{~W}$ transmitting power and $-146 \mathrm{~dB}$ receiving sensitivity is built. Detailed link budget is shown in Table 2. OPNET supports a common wireless channel model and provides an antenna definition tool. Antenna is modeled using the tool, as shown in Fig. 5. 


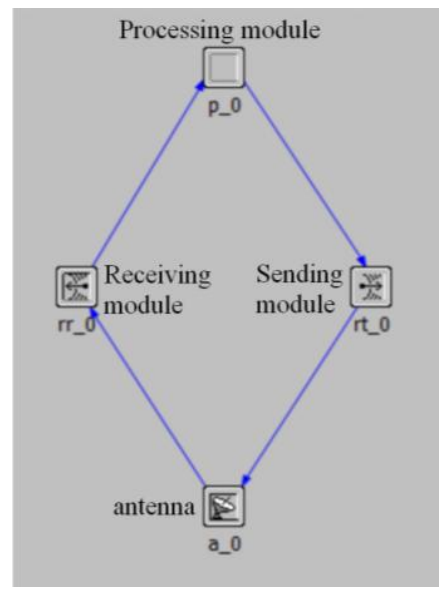

Fig. 4 Node model

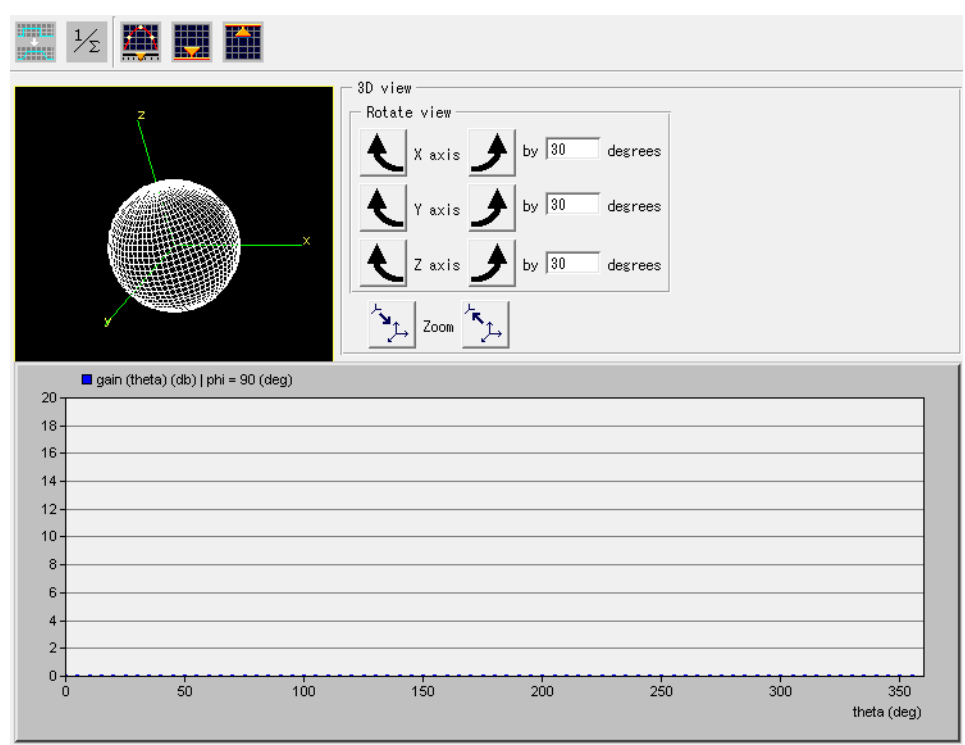

Fig. 5 Antenna model

Table 2 Inter-satellite link budget

\begin{tabular}{|c|c|c|c|}
\hline Item & \multicolumn{2}{|c|}{ Parameter } & Remarks \\
\hline Transmit power $(\mathrm{P})$ & \multicolumn{2}{|c|}{ OdBW } & $1 \mathrm{~W}$ \\
\hline Transmit antenna gain (Gpt) & \multicolumn{2}{|c|}{$0 \mathrm{dBi}$} & \\
\hline Transmitter transmission loss (Lt) & \multicolumn{2}{|c|}{$3 \mathrm{~dB}$} & \\
\hline Launch frequency (f) & \multicolumn{2}{|c|}{$2.4 \mathrm{GHz}$} & $\mathrm{S}$ band \\
\hline Transmission distance $(\mathrm{d})$ & $1 \mathrm{~km}$ & $50 \mathrm{~km}$ & $1-50 \mathrm{~km}$ \\
\hline Space propagation loss (Ls) & $100 \mathrm{~dB}$ & $134 \mathrm{~dB}$ & $\mathrm{Ls}=92.44+20 \lg f+20 \operatorname{lgd}$ \\
\hline Receive antenna gain (Grp) & \multicolumn{2}{|c|}{ OdBi } & \\
\hline Feeder loss $(\mathrm{Lr})$ & \multicolumn{2}{|c|}{$3 \mathrm{~dB}$} & \\
\hline Polarization loss $(\mathrm{Lp})$ & \multicolumn{2}{|c|}{$1 \mathrm{~dB}$} & Linear polarization \\
\hline Receiver sensitivity (Sen) & $-138 \mathrm{dBW}$ & $-146 \mathrm{dBW}$ & Bit error rate $1 \times 10^{-6}$ \\
\hline Channel coding gain $(\mathrm{Gc})$ & \multicolumn{2}{|c|}{$2.4 \mathrm{~dB}$} & Gore code $(23,12,7)$, bit error rate under $1 \times 10^{-6}$ \\
\hline $\operatorname{Margin}(\mathrm{dB})$ & $33.4 \mathrm{~dB}$ & $7.4 \mathrm{~dB}$ & P+GPT-Lt-Ls+Grp-Lr-Lp-Sen+Gc \\
\hline
\end{tabular}

Process model is built according to Fig. 6. As OPNET uses a low-level discrete event simulation mechanism, the process model is build in the form of state machines. It contains 5 states: init, listen, request_in, slave, master. The arrows between states indicate the condition of state transition. Functions of each state is defined using $\mathrm{C}$-like programming language functions, including message receiving, processing, packaging, sending, and state determining.

Finally, the network model including 6 nodes is established, as shown in Fig. 7. OPNET supports space position modeling and the circular radius in Fig. 7 is $25 \mathrm{~km}$.

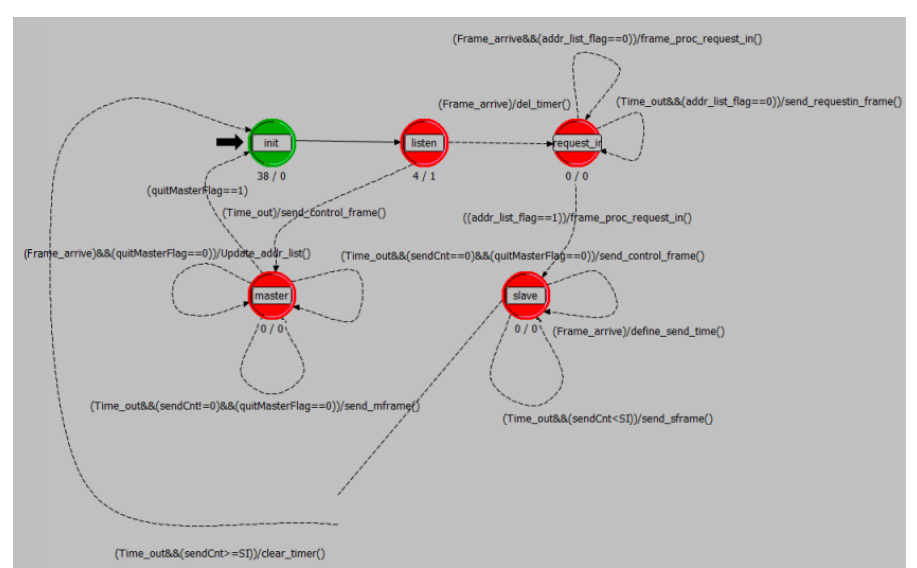

Fig. 6 The process model

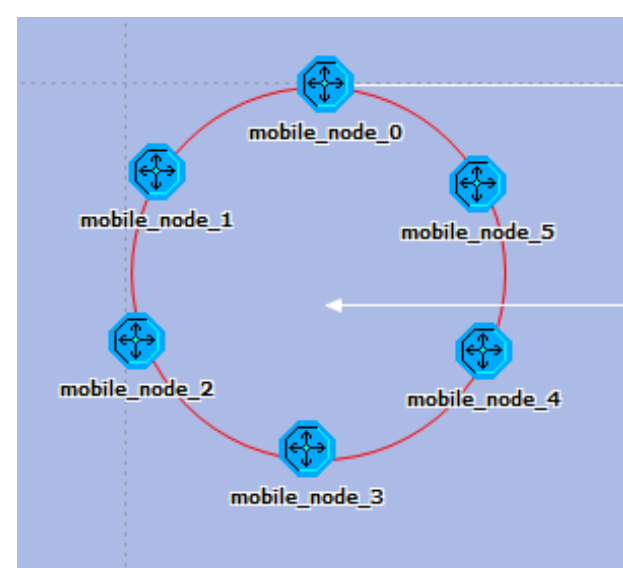

Fig. 7 Network model for 6 nodes 


\section{Simulation of Space Self-organizing Network}

Firstly the networking time is tested under the condition of a fixed node location, as shown in Fig. 8. Networking time is the time from the begin of the simulation to the end of autonomous networking. As can be seen from the test results, average time of six network nodes is 5.6 seconds, which is relatively fast.

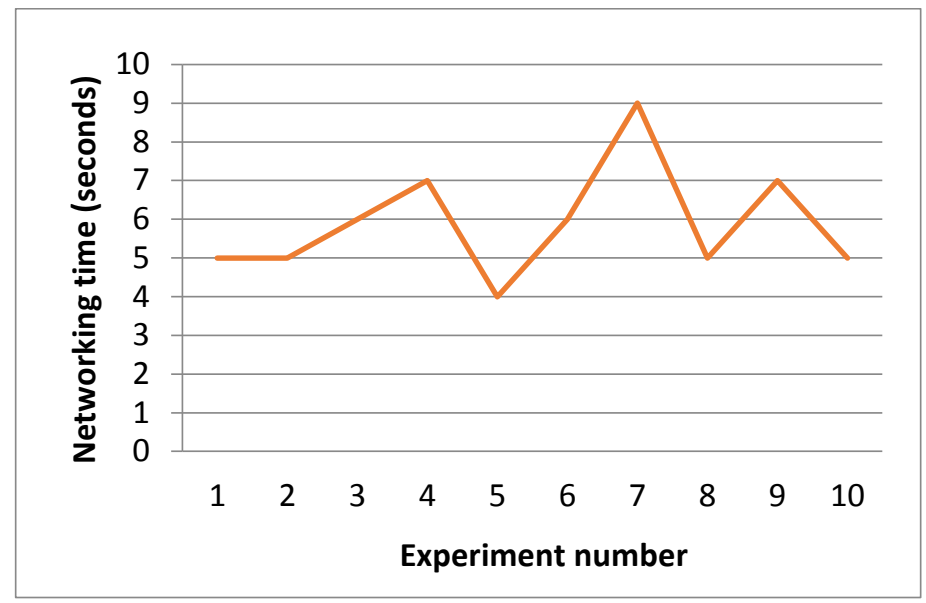

Fig. 8 Networking time

Fig. 9 shows the process of autonomous network setup, node joining, and node exiting. Position of nodes 1-5 stays unchanged while node 0 move in specified path, away from the other nodes and then close. As shown in Fig. 9(b), node 0 goes out of network communication range during the movement and thus leaves the network. Therefore its packets can no longer be received by other nodes. At the same time the other nodes left in the network (nodes 1-5) keep working. As shown in Fig. 9(c), when node 0 gets close back to the network, it meets the communication request and reenters the network. If node 0 is in master mode after network is established, the remaining nodes will be reorganized when node 0 goes far. Fig. 10 shows the process of random movement of six nodes. As can be seen from the test, as long as the node is in the range of wireless communications, autonomous networking process works well and the network is stable and reliable.

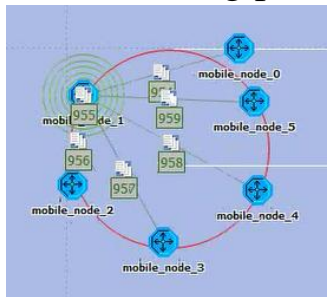

a) networking

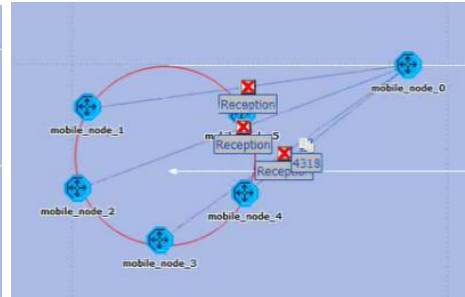

b) node exiting

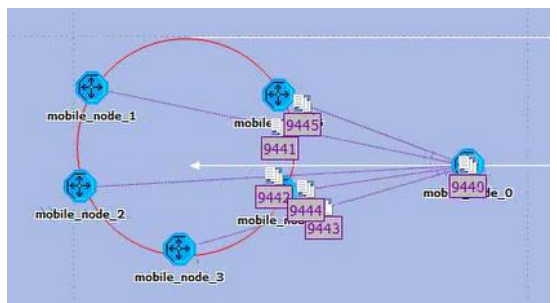

c) node joining

Fig. 9 Autonomous networking and network reorganizing process (node 0 moves in fixed path)

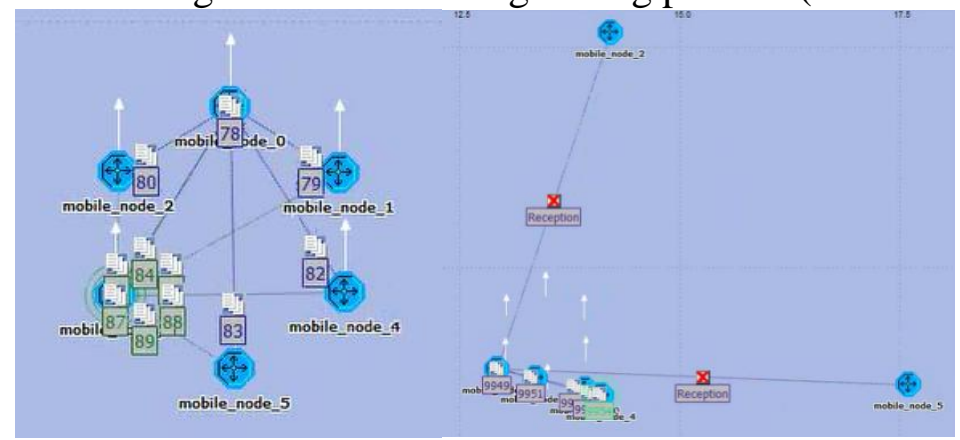

Fig. 10 Autonomous networking and network reorganizing process (each node move randomly)

After the scheme proves to be practical, the protocols are programmed based on the RF SoC and the physical test is carried out as shown in Fig. 11. For TDMA networks, time synchronization is an important index. The time synchronization is measured in physical test. As shown in Fig. 12, each node outputs a pulse through the I/O port at the beginning of the slot. It is very clear that the time slot is very uniform, representing the stability of the network work. 


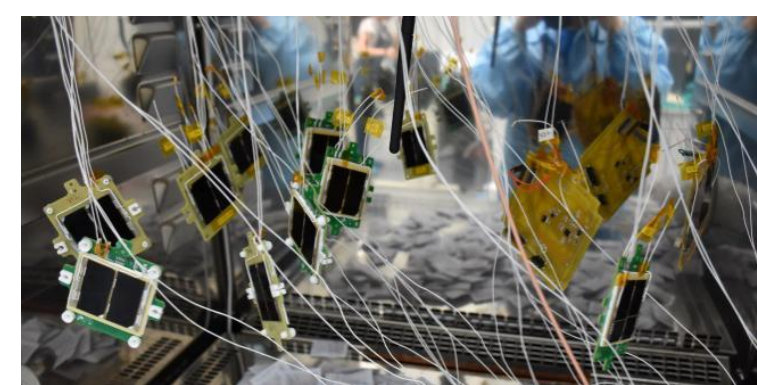

Fig. 11 Multi-node networking test

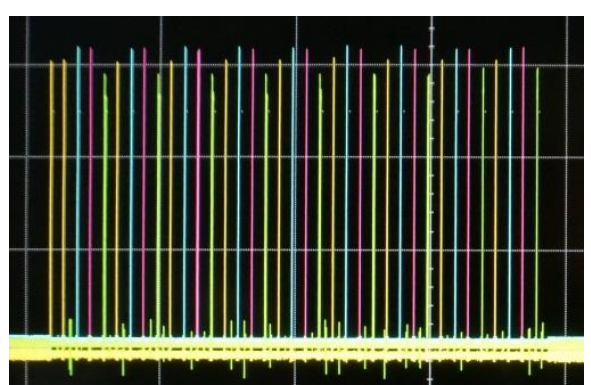

Fig. 12 Time slot measurement

\section{Summary}

A lightweight scheme of space self-organizing network based on TDMA is proposed for cluster flying micro-nano satellites. The network protocol is modeled based on OPNET. Simulation experiments, such as autonomous negotiated networking, nodes joining and exiting, have been carried out and the results show that this scheme is feasible. Protocol programming and physical test are carried out using RF SoC. The results show that the network is stable and reliable. On-orbit tests of space self-organizing network payloads have been carried out successfully in the cluster flying TianTuo-3 satellites, including autonomous networking, indirect telemetry and telecontrol. This paper has a good reference for the design of space self-organizing network and the development of ground simulation experiments.

\section{References}

[1] Brown O. Fractionated space architectures: a vision for responsive space. AIAA 4th Responsive Space Conference, Los Angeles, CA, 2006.

[2] Vladimirova T. Enabling technologies for distributed picosatellite missions in LEO. the First NASA/ESA Conference on Adaptive Hardware and Systems (AHS'06), 2006.

[3] Muri P, McNair J, Antoon J, et al. Topology design and performance analysis for networked earth observing small satellites. MILITARY COMMUNICATIONS CONFERENCE, Baltimore, Maryland, 2011.

[4] Nakamura Y, Faber N, Mauro D, et al. Heterogeneous Spacecraft Networks: Performance analysis for low-cost Earth Observation missions. 2014 IEEE Aerospace Conference, 2014.

[5] Michel T, Thapa B and Taylor S. 802.11s based multi-radio multi-channel mesh networking for fractionated spacecraft. 2013 IEEE Aerospace Conference, 2013.

[6] Bartl M, Hosek J, Matocha T, et al. Integration of real network components into OPNET Modeler Co-simulation process. WSEAS TRANSACTIONS on COMMUNICATIONS, 9(9), 2010: 553 $-562$. 